\title{
Os efeitos do veganismo no desenvolvimento infantil
}

\author{
The effects of veganism on child development \\ Los efectos del veganismo en el desarrollo infantil
}

Recebido: 23/08/2021 | Revisado: 27/08/2021 | Aceito: 27/08/2021 | Publicado: 29/08/2021

\author{
Eleni Alves Cachiado \\ ORCID: https://orcid.org/0000-0001-9204-0402 \\ Centro Universitário Fametro, Brasil \\ E-mail: elennyalves@outlook.com.br \\ José Estelito Soares \\ ORCID: https://orcid.org/0000-0002-9350-9775 \\ Centro Universitário Fametro, Brasil \\ E-mail:joseestelitocs@gmail.com \\ Prissila Corrêa dos Santos \\ ORCID: https://orcid.org/0000-0002-4755-5324 \\ Centro Universitário Fametro, Brasil \\ E-mail: prissilasantos@ hotmail.com \\ José Carlos de Sales Ferreira \\ ORCID: https://orcid.org/0000-0002-1867-8229 \\ Centro Universitário Fametro, Brasil \\ E-mail: jcarlos.sales@gmail.com
}

\begin{abstract}
Resumo
Introdução: Do ponto de vista científico, o veganismo é conhecido por causar muitas deficiências nutricionais, logo, cria-se vertentes sobre quais impactos esse tipo de dieta acarretará na população infantil, se positivo ou negativo. Objetivo: elucidar os impactos do veganismo no desenvolvimento infantil, tanto pontos positivos quanto os negativos, avaliando-os de acordo com a perspectiva nutricional. Metodologia: Para a realização desse estudo optou-se por uma pesquisa na modalidade de revisão integrativa de literatura. A revisão integrativa determina o conhecimento atual sobre uma temática específica, já que é conduzida de modo a identificar, analisar e sintetizar resultados de estudos independentes sobre o mesmo assunto. Resultados e Discussão: Dentre os indivíduos seguintes do estilo de vida vegano, a deficiência de nutrientes é o primeiro impacto negativo mais frequente. Além disso, outro malefício desta forma alimentar é que grande parte dos adeptos necessitam fazer suplementação de nutrientes aditivos alimentares. Conclusão: Através do desenvolvimento desse estudo foi possível analisar e pontuar questões voltadas ao veganismo na infância, bem como seus benefícios, quanto o impacto negativo no desenvolvimento infantil. Dentre os indivíduos seguintes do estilo de vida vegano, a deficiência de nutrientes é o primeiro impacto negativo mais frequente.
\end{abstract}

Palavras-chave: Veganismo; Desenvolvimento infantil; Dieta, Nutrição e saúde.

\begin{abstract}
Introduction: From a scientific point of view, veganism is known to cause many nutritional deficiencies, therefore, it creates perspectives on what impacts this type of diet will have on the child population, whether positive or negative. Objective: elucidate the impacts of veganism on child development, both positive and negative points, evaluating them according to the nutritional perspective. Methodology: To carry out this study, we opted for research in the form of an integrative literature review. The integrative review determines current knowledge on a specific topic, as it is conducted in order to identify, analyze and synthesize results of independent studies on the same subject. Results and Discussion: Among the following individuals from the vegan lifestyle, nutrient deficiency is the first most frequent negative impact. In addition, another harm of this form of food is that most adepts need to supplement food additives with nutrients. Conclusion: Through the development of this study, it was possible to analyze and point out issues related to childhood veganism, as well os its benefits, regarding the negative impact on child development. Among individuals following the vegan lifestyle, nutrient deficiency is the first most frequent negative impact.
\end{abstract}

Keywords: Veganism; Child development; Diet; Nutrition and health.

\section{Resumen}

Introducción: Desde un punto de vista científico, se sabe que el veganismo causa muchas deficiencias nutricionales, por lo tanto, crea perspectivas sobre qué impactos tendrá este tipo de dieta en la población infantil, ya sea positiva o negativa. Objetivo: dilucidar los impactos del veganismo en el desarrollo infantil, tanto positivos como negativos, evaluándolos desde una perspectiva nutricional. Metodología: Para la realización de este estudio se optó por una investigación en la modalidad de revisión integradora de la literatura. La revisión integradora determina el conocimiento actual sobre un tema específico, ya que se realiza con el fin de identificar, analizar y sintetizar 
resultados de estudios independientes sobre el mismo tema. Resultados y discusión: Entre las siguientes personas del estilo de vida vegano, la deficiencia de nutrientes es el primer impacto negativo más frecuente. Además, otro daño de esta forma de comida es que la mayoría de los adeptos necesitan complementar los aditivos alimentarios con nutrientes. Conclusión: A través del desarrollo de este estudio, fue posible analizar y señalar temas relacionados con el veganismo infantil, así como sus beneficios, en cuanto al impacto negativo en el desarrollo infantil. Entre las personas que siguen el estilo de vida vegano, la deficiencia de nutrientes es el primer impacto negativo más frecuente.

Palabras clave: Veganismo; Desarrollo infantil; Dieta; Nutrición y salud.

\section{Introdução}

O Veganismo é uma "Ideologia que defende a não utilização de qualquer produto de origem animal" (Aurélio, 2017). Logo, os adeptos desse movimento se alimentam somente de frutas, legumes, sementes, gorduras vegetais e oleaginosas, abdicando até mesmo de qualquer produto e substância testada em animais (Messina, 2021).

São diversos os motivos que estão atraindo pessoas para o estilo de vida vegano, grande parte desse público adota esse regime baseando sua escolha por julgar ser mais saudável (Rola, 2015). Sendo assim, tem se fortalecido principalmente por na maioria das vezes ser hereditário, ou seja, são pais que buscam na medida do possível ensinar a excluir todas as formas de exploração e crueldade animal (IBOPE, 2018).

Entretanto, acende-se um alerta sobre uma nutrição responsável, afinal é no período da infância que ocorre a maior parte do desenvolvimento humano e nesse apogeu de crescimento deve-se receber quantidades superiores de proteínas e algumas vitaminas fundamentais para massa muscular, conjunto de ossos e cérebro (Baroni et al., 2018).

Do ponto de vista científico, o veganismo é conhecido por causar muitas deficiências nutricionais, logo, cria-se vertentes sobre quais impactos esse tipo de dieta acarretará na população infantil, se positivo ou negativo (Agnoli et al., 2017).

De fato, as crianças podem desenvolver patologias se tratando do contratempo causado por essa falta de nutrientes, como: deficiências nos sistemas neurológicos e motores, convulsões, apneias centrais, desnutrição e problemas infecciosos, esses devido as carências nutricionais principalmente das vitaminas B12 e D3, Cálcio e Ácido docosaexaenoico (DHA) e ferro Heme (Simonson, 2019).

Por outro lado, a nutrição vegana quando empregada de forma assertiva oferece benefícios a saúde, esses que são essenciais na prevenção de algumas doenças, como: o diabetes, obesidade e alguns tipos de câncer. E ainda, possibilita uma maior ingestão de vitaminas, fibras e antioxidantes (Melina, 2016).

Há evidências de que dietas desequilibradas, sejam elas veganas ou não, favorecem o aparecimento de patologias que se repercutem no crescimento e desenvolvimento da criança, e que adquirem expressão na vida adulta, nesse contexto, vem à tona a importância de compreender este assunto, para que seja possível enraizar uma alimentação adequada fornecendo o que o corpo precisa (BBC, 2021).

Perante a necessidade de debater a temática atual, o presente estudo busca elucidar os impactos do veganismo no desenvolvimento infantil, tanto pontos positivos quanto os negativos, avaliando-os de acordo com a perspectiva nutricional.

\section{Metodologia}

Para a realização desse estudo optou-se por uma pesquisa na modalidade de revisão integrativa de literatura. A revisão integrativa determina o conhecimento atual sobre uma temática específica, já que é conduzida de modo a identificar, analisar e sintetizar resultados de estudos independentes sobre o mesmo assunto (Silveira et al., 2008).

A Revisão Integrativa é um método de pesquisa apontado como ferramenta de grande relevância no campo da saúde, por proporcionar a busca, a avaliação crítica e a síntese de evidências sobre um tema investigado. Esses aspectos facilitam a identificação dos resultados relevantes, de lacunas que direcionam para o desenvolvimento de futuras pesquisas e auxiliam o profissional a escolher condutas e a tomar decisões, proporcionando um saber crítico (Souza, Silva \& Carvalho, 2010). 
Para extrair os dados dos artigos selecionados, será necessária a utilização de um instrumento previamente elaborado capaz de assegurar que a totalidade dos dados relevantes seja extraída, minimizar o risco de erros na transcrição, garantir precisão na checagem das informações e servir como registro (Souza, Silva \& Carvalho, 2010).

A coleta das informações para a pesquisa bibliográfica será por meio da exploração da base de dados da Biblioteca Virtual em Saúde (BVS), Biblioteca Cientifica Eletrônica Virtual (SCIELO) e Literatura Latino - Americana e do Caribe em Ciências da Saúde (LILACS).

A busca na base de dados será orientada pelos descritores: Veganismo, Desenvolvimento infantil, Dieta, nutrição e saúde, e será realizado em todos os índices, buscando captar o maior número de artigos publicados no período proposto que abordem a temática em discussão.

Para uma análise crítica e reflexiva dos estudos incluídos na revisão, será realizada uma leitura minuciosa e criteriosa destacando os que atingirem os critérios de inclusão e que contemplarem o objetivo proposto, para viabilizar o resultado da pesquisa de forma clara e objetiva.

Serão incluídos os artigos publicados em língua portuguesa nos últimos cinco anos, com texto completo, disponível on-line, com acesso livre. Serão excluídos da amostra os artigos publicados em línguas estrangeiras, os que não apresentarem o texto na integra, artigos que não apresentavam relação direta com o tema, resumos, monografias, dissertações, teses e artigos repetidos.

\section{Resultados e Discussão}

\subsection{O veganismo e se impacto no desenvolvimento infantil}

O veganismo é um movimento de libertação animal em todos os sentidos, baseado na escolha de tentar eliminar todas as formas de exploração e maus tratos. Além de estar relacionado a diversos aspectos sociais, tais como: o direito dos animais, sustentabilidade e o meio ambiente (The Vegan Society, 2017).

Esse estilo de vida surgiu em 1944 quando um pequeno número de pessoas se desmembrou da Sociedade Vegetariana de Leicester, na Inglaterra, optando por não consumir laticínios, ovos ou quaisquer outros produtos de origem animal, iniciando a Sociedade Vegana (Fuller, 2016).

Entretanto, não se limitou apenas a ser uma forma de se alimentar, o veganismo ficou marcado por ser uma filosofia de vida, no qual se repudia todas as frentes possíveis, incluindo mercado, trabalho forçado, entretenimento ou qualquer atividade que o tire o animal do seu habitat ou possa lhe causar desconforto, se distinguindo totalmente do vegetarianismo que é apenas uma dieta de alimentar (Ferrigno, 2016).

Logo, esse tipo de alimentação é considerado por muitos uma dieta saudável justamente por não conter processados que são ditos maléficos para saúde. Entretanto, há uma série de nutrientes que são tirados e que grande parte deles são difíceis de encontrar em produtos de origem vegetal, podendo acarretar patologias (BBC, 2016).

Nesse sentido, temos como as principais deficiências nutricionais a falta de: ômega-3, os ácidos graxos, ferro, zinco, cálcio e vitaminas D e B12, principalmente por serem de origem animal, logo necessitando de suplementação e acompanhamento médico especializado (Oswaldo Cruz, 2020).

Um estudo publicado no Journal of Agricultural and Food Chemistry (2019) mostrou que pessoas que seguem o estilo de vida vegano tendem a ter níveis elevados de homocisteína no sangue e diminuição dos níveis de HDL, o "bom" colesterol, resultando em um risco elevado de desenvolver coágulos de sangue ou endurecimento das artérias, condições que podem levar a ataques cardíacos e acidente vascular cerebral (AVC).

Portanto, quando essa adaptação alimentar é realizada em crianças, os perigos são maiores, pois o déficit desses nutrientes contribui seguramente para o crescimento e desenvolvimento, considerando que nesta fase a alimentação deve ser 
rica em macro e micronutrientes. Contudo, é possível adquirir essa dieta na infância consumindo apenas alimentos de origem vegetal, contanto que tenha acompanhamento pediátrico e nutricional (Pinho et al., 2016).

\subsection{Os benefícios da alimentação vegana na infância}

O início alimentar de uma criança ocorre no segundo semestre de vida, como forma de suprir a demanda energética e nutricional apropriada até que a mesma consiga ser inserida na dieta familiar (Ali, 2016).

A European Society for Paediatric, Gastroenterology, Hepatology and Nutrition (ESPGHAN), a American Dietetic Association (ADA), a Academy of Nutrition and Dietetics (AND) e a American Academy of Pediatrics (AAP) afirmam que, embora haja uma escassez de informações sobre introdução alimentar vegana, quando bem elaborada, as necessidades nutricionais conseguem ser contempladas, sem prejuízo ao desenvolvimento cognitivo e ao ganho ponderal da criança. Entretanto, a ESPGHAN adverte que ajustes são fundamentais, além de uma supervisão e orientação médicas rigorosas (Biarge, 2018).

Contudo, por conta do alto teor de fibras, fitoquímicos e antioxidantes consumidos em uma alimentação baseada em vegetais, cereais de grãos integrais, frutas, nozes, leguminosas, entre outros, é possibilitado vantagens clínicas importantes durante infância, como um menor risco para hipertensão, obesidade, e ainda, diabetes com início na fase adulta (Pimentel, 2018).

Assim como, a redução em $40 \%$ do risco de desenvolver algum cancro, devido a exclusão de alimentos de origem animal. Desta forma o sistema imune estará mais forte e preparado para combater células cancerígenas, além de melhorar trato digestivo, e saúde ósseas (The Guardian, 2016).

\subsection{Os impactos negativos do veganismo no desenvolvimento infantil}

Dentre os indivíduos seguintes do estilo de vida vegano, a deficiência de nutrientes é o primeiro impacto negativo mais frequente. Além disso, outro malefício desta forma alimentar é que grande parte dos adeptos necessitam fazer suplementação de nutrientes aditivos alimentares. Sendo assim, quando aderida na fase infantil deve-se ter uma dieta planejada corretamente para que possa evitar o desencadeamento de patologias graves e atrapalhar o desenvolvimento da criança (Andres, et al., 2016). Como supracitado, é comum que ocorra a falta de nutrientes quando se adere a alimentação vegana durante a infância. Nos quais são: deficiência de vitamina B12, vitamina D, de ferro, cálcio.

\subsubsection{Deficiências nutricionais - Vitamina B12}

A vitamina B12, ou cianocobalamina desempenha funções como a manutenção do metabolismo do sistema nervoso, a formação do DNA e a produção de glóbulos vermelhos saudáveis para o sangue, e sua fonte natural restringe-se apenas a alimentos de origem animal, especialmente leite, carne e ovos (Herrmann et al, 2017).

Por isso, crianças com a alimentação vegana devem consumir a vitamina B12 por meio de alimentos fortificados, ou seja, a indústria adiciona artificialmente em produtos como soja, carne de soja e cereais matinais ou até mesmo através do uso de suplementos (Graham et al. 2016).

Tendo em vista que durante a infância o corpo está em desenvolvimento a falta da vitamina pode contribuir silenciosamente para problemas desde os de ordem sensoriais até os distúrbios da aprendizagem, além de ocasionar transtornos hematológicos, e cardiovasculares, e o desenvolvimento de patologias cerebrais de diferentes graus de severidade, podendo até mesmo tornarem-se irreversíveis (Andres et al., 2016). 


\subsubsection{Deficiências nutricionais - Vitamina D}

A vitamina D, é presente no reino animal, sendo praticamente escassa no reino vegetal, por esse motivo é necessário buscar meios para mantê-la na dieta alimentar na quantidade correta, contudo, é possível obtê-la apenas em alimentos fortificados, como leite de soja e cereais e também em cogumelos (Hovinen, 2021).

Por possuir função hormonal, quando seus níveis estão reduzidos no sangue, pode haver maior possibilidade de desenvolvimento de algumas alterações, como dor e fadiga muscular, hipertensão arterial, doenças cardiovasculares, entre outras (SBP, 2021).

\subsubsection{Deficiências nutricionais - Ferro}

O ferro é um micronutriente que exerce diversas funções metabólicas. A principal fonte alimentar desse mineral é a carne vermelha. Na deficiência de ferro a anemia ferropriva é a forma mais grave decorrente da privação dentro do organismo levando à uma diminuição da produção, tamanho e teor de hemoglobina dos glóbulos vermelhos, hemácias. Em crianças os sintomas mais frequentes relatados são fadiga crônica, desmaios, apatia, anorexia, diminuição da capacidade física e dificuldade na concentração e lapsos de memória. Alguns estudos relatam diminuição da função imunológica, com aumento da frequência e duração das infecções (Braga, 2011).

Salienta-se também que a anemia ferropriva pode implicar diretamente no progresso dos efeitos motores e processos mentais durante a infância, além de comprometer no aprendizado e no rendimento escolar (Rivera, 2016).

\subsubsection{Deficiências nutricionais - Cálcio}

$\mathrm{O}$ crescimento de o crescimento de uma criança, consumir a quantidade correta de cálcio influenciará diretamente no seu desenvolvimento ósseo, tornando-se o principal micronutriente para a manutenção da estrutura dos ossos na vida adulta (Zhu \& Prince, 2016).

Estudos mostram que na infância é necessário consumir de duas a quatro vezes mais cálcio por kg de peso em relação aos adultos (Leão \& Santos, 2017). Quando a ingestão é insuficiente acarreta prejuízos na evolução genética (altura genética) e na massa óssea (Pettifor, 2017).

No caso de crianças que segue o estilo de vida vegano, essa falta pode ocorrer devido a maior concentração desse micronutriente está presente no leite de vaca e búfala, ambos de origem animal, e, por conseguinte seus derivados, mas também pode ser encontrada em outras fontes como carnes de peixe e frutos do mar. Contudo, também é possível consumir o cálcio nas verduras verdes, exemplo: brócolis, folhas de mostarda e a couve, entretanto, na sua quantidade é insuficiente comparado ao leite (Pereira, 2016).

\section{Conclusão}

Através do desenvolvimento desse estudo foi possível analisar e pontuar questões voltadas ao veganismo na infância, bem como seus benefícios, quanto o impacto negativo no desenvolvimento infantil.

Dentre os indivíduos seguintes do estilo de vida vegano, a deficiência de nutrientes é o primeiro impacto negativo mais frequente. Além disso, outro malefício desta forma alimentar é que grande parte dos adeptos necessitam fazer suplementação de nutrientes aditivos alimentares. Sendo assim, quando aderida na fase infantil deve-se ter uma dieta planejada corretamente para que possa evitar o desencadeamento de patologias graves e atrapalhar o desenvolvimento da criança. 


\section{Referências}

Abonizio, J. (2016). Conflitos à mesa: vegetarianos, consumo e identidade. Revista Brasileira de Ciências Sociais, Cuiabá, 31(90), $115-137$.

Agnoli, C., et al. (2017). Position paperon vegetarian diets from the working group of the Italian Society of Human Nutrition. Nutrition, Metabolism and Cardiovascular Diseases, New York, 27(12), 1037-1052.

Ali, A. B. G., Andrade, B. C. P., Oliveira, M. M. S., \& Kashiwbara, T. G. B. (2016). Introdução alimentar em crianças veganas - Revisão de literatura. Ver Uningá Review, 20(3), 82-87.

Andres, E., et al. (2016). Vitamin B12 (cobalamin) deficiency in elderlypatients. CMAJ, 171(3), 251-9.

Appleby, P., \& Key, T. (2016). The long-term health of vegetarians and vegans. Proceedings of The Nutrition Society, Cambridge, 75(3), 287-293.

Armelagos, G. (2016). Brain evolution, the determinates of food choice, and the omnivore's dilemma. Critical Reviews in Food Science and Nutrition, 54(10), $1330-1341$.

Aurelio. https://www.dicio.com.br/veganismo/.

Baroni, L., Goggi S., Battaglino, R., Berveglieri, M., et al. (2018). Vegannutrition for mothersandchildren: practical tools for healthcare providers. Nutrients.

Burns-Whitmore B., Haddad E., Sabate J., \& Rajaram S. (2016). Effects of supplementing n-3 fatty acidenriche deggs and walnutson cardiovascular disease risk markers in healthy free-living lacto-ovo-vegetarians: a randomized, crossover, free-living intervention study. Nutr J. 13:29.

BBC NEWS BRASIL. (2016).

Biarge, M. M. (2018). Ninõs vegetarianos, ¿ninõssanos? Curso de Actualización Pediatría. Madrid: Lúa Ediciones, 3(2), 23-234.

Braga, J. A. P., Barbosa, T. N. N., Ferreira, A. M., In: Palma D., Oliveira, F. L. C., Escrivão, M. A. M. S, et al. (2017). Guia de Nutrição Clínica na Infância e na Adolescência. Barueri, São Paulo: Manole, 219-41.

Chauveau, P., et al. (2016). Vegetarianism: advantages and drawbacks in patients with chronic kidney diseases. Journal of Renal Nutrition, New York, 23(6), $399-405$.

Crowe, F., et al. (2016). Plasma concentrations of 25-hydroxyvitamin D in meat eaters, fish eaters, vegetarians and vegans: results from the EPIC-Oxford study. Public Health Nutrition, Cambridge, 14(2), 340-346.

Dinu, M., et al. (2017). Vegetarian, vegan diets and multiple health outcomes: a systematic review with meta-analysis of observational studies. Critical Reviews in Food Science and Nutrition, 57(17), 3640-3649.

Dror, D. K., \& Allen, L. H. (2018). Effect of vitamin B12 deficiency on neurodevelopment in infants: current knowledge and possible mechanisms. Nutrition Reviews, 66(5), 250-255.

Dagnelie N. D. (2016). Nutrition and health-potential health benefitsan drisk sof vegetarianism and limited consumption of meat in the Netherlands. Ned Tijdschr Geneeskd. 147(27), 1308-13.

Elorinne A. L., Alfthan G., Erlund I., Kivimaki H., Paju A., Salminen I., et al. (2016). Food and nutriente in take and nutritional status off in Nish vegans and non-vegetarians. PLoSOne, 11(2).

Elmadfa, I., \& Singer, I. (2016). Vitamin B-12 and homocysteine status among vegetarians: a global perspective. The American Journal of Clinical Nutrition, $89(5), 1693 \mathrm{~S}-1698 \mathrm{~S}$.

Ferrigno, M. (2016). Veganismo e libertação animal: um estudo etnográfico. Dissertação de Mestrado. Universidade Estadual de Campinas.

Fuller, J. (2016). The cultural creation of affect amongst vegan consumers: Aneth no graphic analysis of online consumption communities. Department of Service Management and Service Studies, Lund University.

Graham, S. M., et al. (2016). Long-term neurologic consequences of nutritional vitamin B12 deficiency in infants. J Pediatr, $121(05), 710-4$.

Gil, A. C. (2011). Métodos e Técnicas de Pesquisa Social. (6ª . Ed.): Atlas.

Herrmann, W., et al. (2016). Vitamin B-12 status, particularlyholotranscobalamin II andmethylmalonicacidconcentrations, andhyperhomocysteinemia in vegetarians. Am J Clin Nutr, 78(1), 131-6.

Hovinen, T., et al. (2021). Vegan diet in Young children remodels metabolism and challenges the status esofessential nutrients. EMBO Mol Med, e13492.

Hyde, N., et al. (2017). Maternal nutrition during pregnancy: intake of nutrients important for bone health. Maternal and Child Health Journal, 21(4), 845851.

IBOPE. Pesquisa do IBOPE aponta crescimento histórico no número de vegetarianos no Brasil. IPSOS.

Institut National De Prévention Et D'éducation Pour La Santé.(2015). Guide nutrition de la grossesse. Paris: Inpes, 2015. https://www.mangerbouger.fr/pro/IMG/pdf/Livret_accompagnement_grossesse.pdf.

Instituto Brasileiro De Opinião Pública E Estatística. (2018). Pesquisa de opinião pública sobre vegetarianismo. http://www.svb.org.br/images/Documentos/JOB_0416_VEGETARIANISMO.pdf. 
Jiménez-Aguilar, A., Gaona-Pineda, E., Mejía-Rodríguez, F., Gómez-Acosta, L., Humarán, I., \& Flores-Aldanam. (2016). Consumption of fruits and vegetable sand health status of Mexican children from the National Health and Nutrition Survey.

Lampe, J. W. (2017). Health effectsofvegetablesandfruit: assessingmechanismsofaction in human experimental studies. Am J Clinnutr, 70(3 Suppl):475S490S.

Leão, A. L. M., \& Santos, L. C. Consumo de micronutrientes e excesso de peso; existe relação? Rev. bras. epidemiol. São Paulo. 15(1), 85-95.

Lopes, M. S., et al. (2016). Iodo e tireoide: o que o clínico deve saber. Revista Científica da Ordem dos Médicos, Lisboa, 3(25), 174-178.

Melina, V., Craig, W., \& Levin, S. (2016). Position oftheAcademyofNutritionandDietetics: Vegetarian Diets. Journal of the Academy of Nutrition and Dietetics: 116(12).

Messina, V. (2021). Considerations in planningvegan diets: Children.

Pereira, G. A. P., et al. (2016). Cálcio dietético: estratégias para otimizar o desenvolvimento infantil. Rev. Bras. Cresc. e Desenv. 21(21), 156-171.

Moran, V. H., et al. (2016). The relationship between zinc intake and serum/plasma zinc concentration in pregnant and lactating women: a systematic review with dose-response meta-analyses. Journal of Trace Elements in Medicine and Biology, 26, 74-79.

Nguyen, V. Q., et al. (2016). Prevalence and correlates of zinc deficiency in pregnant Vietnamese women in Ho Chi Minh City. Asia Pacific Journal of Clinical Nutrition, 22(4), 614-619.

Pawlak, R., et al. (2017). How prevalent is vitamin B12 deficiency among vegetarians? Nutrition Reviews, 71, 110-117.

Piccoli, G. B., et al. (2016). Vegan-vegetarian diets in pregnancy: danger or panacea? A systematic narrative review. BJOG: an International Journal of Obstetrics and Gynaecology, 122(5), 623-633.

Perry, C. L., Mcguire, M. T., Neumark-Sztainer, D., \& Story, M. (2016). Characteristics of vegetarian adolescents in a multi ethnic urban population. $J$ Adolesc Health, 29(6), 406-16.

Pettifor, J. M., et al. (2016). Metabolism in Children in Developing Countriens. Ann NutrMetab, 64(supl 2), 15- 22.

Pimentel D., Tomada I., \& Rêgo C. (2018). Alimentação vegana nos primeiros anos de vida: considerações e orientações [trabalho de conclusão de curso]. Porto: Universidade Católica Portuguesa.

Pinho, et al. (2016). Guia Alimentar Mediterrânico: Relatório justificativo do seu desenvolvimento. Programa Nacional para a Promoção da Alimentação Saudável. Direção-Geral da Saúde.

Pistollato, F., et al. (2016). Plant-based and plant-rich diet patterns during gestation: beneficial effects and possible shortcomings. Advances in Nutrition, 6, 581-591.

Rogne, T., et al. (2017). Associations of maternal vitamin B12 concentration in pregnancy with the risks of preterm birth and low birth weight: a systematic review and meta-analysis of individual participant data. American Journal of Epidemiology, 185(3), 212-223.

Rivera, J. A., Hotz, C, González-Cossio, T., Neufel, D. L., \& Garcia - Guerra A. (2013). The effect of micronutrient deficiencies on child growth: a review of results from community-based supplementation trials. J Nutr. 133(11 Suppl 2):4010S-4020S.

Rola, C. (2015). Vegetarianismo e comportamento alimentar: comportamentos alimentares disfuncionais e hábitos alimentares em dietas vegetarianas. 2015. 103 f. Dissertação (Mestrado em Doenças Metabólicas e Comportamento Alimentar) - Universidade de Lisboa, Lisboa.

Simonson, W. (2019). Shouldvitamin C routinelybegivenwith oral iron supplements? "É seguro para o bebê manter uma dieta sem alimentos de origem animal já desde os primeiros meses? Há algum risco envolvido na opção?” Sociedade brasileira de pediatria. geriatrnurs, 40(3): 327-328.

Wood, K. E., Mantzioris, E., Gibson, R. A., Ramsden, C. E., \& Muhlhausler, B. S. (2015). The effect of modifying dietary LA and ALA intakeson omega-3 long chain polyunsaturated fattyacid (n-3 LCPUFA) status in human adults: a systematic review and commentary. Prostag Leukot rEss, 95:47-55.

Zhu, K., \& Prince, R. L. (2016). Calciu mand bone. ClinBiachem, 9, 936-942. 